\title{
A Transmission Electron Microscopy Study on Self-Catalyzed GaAs Nanostructures
}

\author{
Young Heon KIM, Dong Woo PARK, Sang Jun LEE and Sang Jung AHN \\ Korea Research Institute of Standards and Science, Daejeon 305-340, South Korea
}

Semiconductor nanostructures are potential building blocks for many applications in photonics, electronics, physics, and life sciences. They are also a good candidate to study quantum mechanics and related novel phenomena, based on the dimensionality and the size reduction. Among various nanostructures, one-dimensional (1-D) III-V nanostructures grown on Si substrates have received a lot of attention, due to the excellent physical properties of being a direct bandgap, combined with the wellestablished Si technology [1].

However, there are a number of fundamental questions to challenge, regarding the microstructural property in realizing the actual device based on 1-D nanowires (NWs) because of several barriers. Most of the reported 1-D nanostructures have been grown using a vapor-liquid-solid (VLS) growth mechanism. The use of a catalyst in the growth of nanostructures can cause problems like unintentional doping and the elimination of the catalyst for the device fabrication. Therefore, a catalyst-free method is considered to be the best solution for the growth of applicable 1-D nanostructures. The successful growth of catalyst-free 1-D III-V nanostructures has been reported as using a thin oxide layer on $\mathrm{Si}$ substrates [2]. However, among III-V nanostructures, arsenide (As)-based compound semiconductors show the existence of uncontrolled polytypism related to defective structures, such as twin planes and stacking faults [3]. Although a cubic zinc-blende (ZB) structure is adopted in bulk form as a stable phase, the hexagonal wurtzite (WZ) structure is frequently observed in As-based compound nanostructures. Although the defect behaviors and the polytypism of III-V nanostructures have been demonstrated from theoretical viewpoints and based on kinetic theories by many authors, the effort to understand those phenomena is insufficient, since, the control of crystal structures and defects related to the phase evolutions is one of the critical challenges of 1-D III-V nanowires.

In this talk, we present detailed investigation on the microstructural properties of 1-D GaAs nanostructures grown on a Si (111) substrate by a catalyst-free method. The microstructural properties of GaAs nanostructures were discovered by (scanning) transmission electron microscope ((S)TEM) techniques in order to evaluate the defect behavior and the interface structure. Specifically, the atomic arrangements at the interfaces were studied using high-angle annular dark field (HAADF) and annular bright field (ABF) micrographs taken by spherical aberration (Cs)-corrected STEM. In addition, energydispersive X-ray spectroscopy (EDS) and electron energy-loss spectroscopy (EELS) were used to address compositional variations $[4,5]$.

A quantitative analysis of the EDS and EELS results showed that the round droplet at the top of the nanostructures was pure gallium (Ga) (Figure 1). The arsenic (As) element was detected by passing the interface between the $\mathrm{Ga}$ droplet and the GaAs nanostructure in the EELS line profile. Atomic arrangements near the $\mathrm{Ga}$ droplet/GaAs nanostructure interfaces were complex and irregular. The contrast of dark-field and bright-field images is opposed to each other (Figure 2). Although the nanostructure is thick, the dark-field image clearly shows the image contrast and it appears to be symmetric at both side edges along the growth direction of the nanostructure. In addition, fringes 
vertically aligned along the growth direction, caused by crystalline defects such as the twin boundaries and stacking faults, are more clearly observed in the dark-field image than in the bright-field image.

Finally, we demonstrate the phase transition and the gallium $(\mathrm{Ga})$-droplet behaviors dependent on the shapes and the growth conditions of 1-D GaAs nanostructures.

\section{References:}

[1] M.S. Gudiksen, L.J. Lauhon, J. Wang, D.C. Smith, C.M. Lieber, Nature (2002), 415, 617.

[2] B. Mandl, J. Stangl, T. Mårtensson, A. Mikkelsen, J. Eriksson, L. Karlsson, G. Bauer, L. Samuelson and W. Seifert, Nano Lett.(2006), 6, 1817.

[3] F. Glas, J. Harmand and G. Patriarche, Phys. Rev. Lett. (2007), 99, 146101.

[4] Y.H. Kim, D.W. Park and S.J. Lee, Appl. Phys. Lett. 100 (2012), 033117.

[5] Y.H.Kim, D.W. Park, S.J. Lee, K. Kim and Y.C. Park, Appl. Phys. Lett. 100 (2012), 133112.

[6] This research was supported by Nano.Material Technology Development Program through the

National Research Foundation of Korea (NRF) funded by the Ministry of Education, Science and

Technology. Work in part supported by the Korea Research Council of Fundamental Science and Technology (KRCF) through a Basic Research Project managed by the Korea Research Institute of Standards and Science (KRISS).

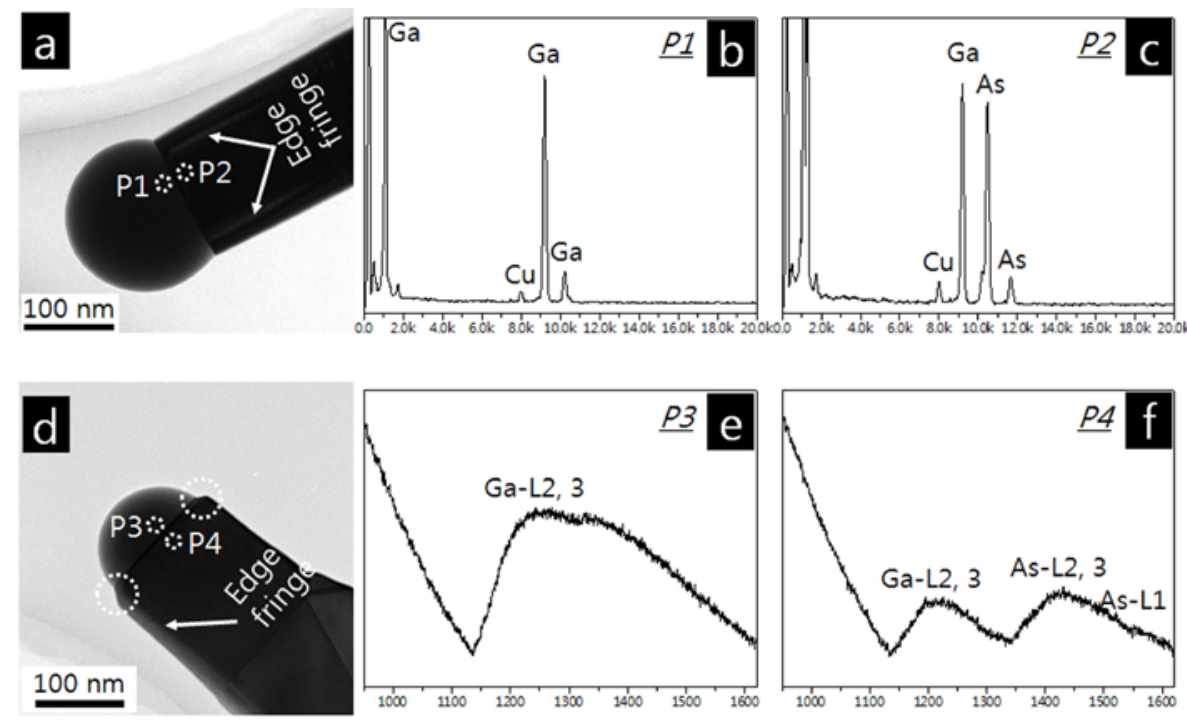

Figure 1. (a) bright-field (BF) TEM image of a hexagonal-shaped 1-D GaAs nanostructure. (b and c) EDS elemental spectra obtained from the points I (P2) and II (P2) in (a), respectively. (d) BF TEM image of a pentagonal-shaped 1-D GaAs nanostructure. (e and f) EELS spectra obtained from the points III (P3) and IV (P4) in (c), respectively.
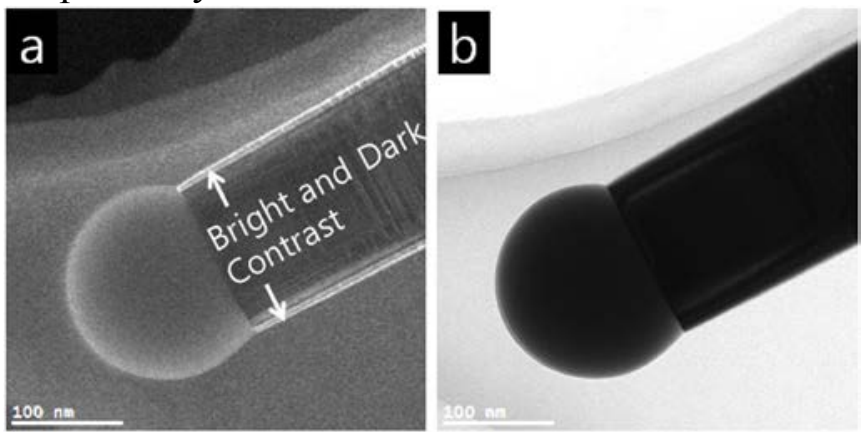

Figure 2. Dark-field (a) and bright-field (b) TEM images of a typical hexagonal nanostructure. 